\title{
1945 als Zäsur? Einige Zeitschriften der ersten Stunde mit Merkur und Joachim Moras im Mittelpunkt
}

\section{5 as a caesura? Several early post-war magazines with Merkur and Joachim Moras in the center of attention}

\author{
Aleš Urválek
}

\begin{abstract}
The paper introduces the topic of the lecture series and outlines the problem areas to which the cycle sought an answer. It briefly presents the program of several magazines of the first post-war years (Wandlung, Frankfurter Hefte, Der Ruf), then focuses on the program statements of the magazine Merkur by interpreting them from contemporary contexts. Finally, he presents one of the publishers of the magazine, Joachim Moras, whose projects from 19471961 demonstrate that post-war research cannot be carried out without a network analysis of "longer-lasting" continuities and discontinuities, at least of the period 1930-1960.
\end{abstract}

\section{Keywords}

caesura; magazines; tradition; modern; continuity; encyclopedia; annual 


\section{Einleitend zum Thema der Vorlesungsreihe}

Stunde Null, Jahr Null, Nullpunkt, Kahlschlag, Tabula rasa, Neubeginn, Neuanfang oder aber Anschluss an die Tradition, Rückkehr, Wiederherstellung, wohl auch Restauration, Zwischen gestern und morgen: All diese Begriffe, Metaphern, Buchtitel stecken in ihrer bewusst zugespitzten Gegenüberstellung beide Pole des Feldes ab, auf dem in folgenden Beträgen des ersten Blocks die ersten Nachkriegsjahre bis etwa 1960 eben aus der Spannung zwischen Neuanfang und Wiederanschluss, oder, moderner, Diskontinuität und Kontinuität heraus interpretiert werden. Da diese Problemlage dieses Mal, um die sattsam bekannten Metaphern zu meiden, auf die Frage zugespitzt wurde, inwiefern das Jahr 1945 für die deutschsprachige Kultur eine Art Zäsur dargestellt hat, sei einführend vom Begriff Zäsur auszugehen.

Auf diesen Begriff pflegt man zurückzugreifen, wenn man einen womöglich qualitativen Unterschied zwischen zwei zeitlich eben dadurch zu trennenden Zuständen, Zeiten festhalten und hervorheben will: der Zeit vor der Zäsur sowie der Zeit nach der Zäsur. Dadurch ist dieser als Zäsur erkannte Zustand zugleich imstande, zwei voneinander unterschiedliche Epochen, besser, „den Endpunkt einer älteren und den Anfangspunkt einer jüngeren Epoche ${ }^{\text {" } 1}$ zu konstituieren. In gewissem Sinne vollzieht somit, wer durch das Setzen der Zäsur zwei grundlegend andere Zeiten entstehen lässt, eine überaus ambivalente Tat. Mit autoritativer Gewalt und jäher Plötzlichkeit negiert er, ja tötet, die alte, somit als unbrauchbar bezeichnete Epoche, um durch den selben Akt eine neue, bessere aus der Taufe zu heben. Wer heute in dem Jahr 1945 eine derartige Zäsur erblickt, folgt damit der Sicht derjenigen, die damals, um von Neuem beginnen zu können, die alte Zeit möglichst zum Verschwinden zu bringen, oder vergessen zu machen, bestrebt waren. Ein reiner Tisch als Voraussetzung für den Neuanfang wäre wohl die passende Umschreibung für diese Gemütslage, die Zeitschriftentitel wie Aufbau, Alternative, Gegenwart auf ihre Art auf den Punkt bringen. Wenn nun versuchsweise der idealtypische deutschsprachige Intellektuelle erfasst werden sollte, der allen Grund gehabt hätte, 1945 als Zäsur zu denken (inszenieren), dann hätte man sich ihn als einen vorzustellen, der sich die Attribute der jungen Generation zuschreiben, ${ }^{2}$ seine wichtigsten Lebensjahre weniger mit Studium denn vielmehr an der Front zugebracht haben, seine intellektuelle Etablierung womöglich mit dem Aufbruch einer neuen Zeit zusammenbringen, der also hohe Erwartungen an Gegenwart und Zukunft stellen, die Vergangenheit für geschichtlich überholt, oder aus anderen Gründen für belanglos halten würde. Mit der naheliegenden Folgerung, dass sämtliche Vergangenheiten eher selektiv wahrgenommen, distanziert oder als Anlass zur grundlegenden Metanoia betrachtet werden, deren

1 Schöning, Matthias (2015): „Zäsur“. Probleme einer historiographischen Angewohnheit. In: Meierhofer, Christian - Wörner, Jens (hrsg.): Materialschlachten. Der Erste Weltkrieg und seine Darstellungsressourcen in Literatur, Publizistik und populären Medien 1899-1929. Osnabrück: Universitätsverlag, S. 68.

2 Was mit dem realen Alter wenig zu tun hat, vielmehr geht es darum, durch diesen Akt die symbolischen Vorteile der jungen Generation für sich in Anspruch zu nehmen. Bekanntlich hatten sich etwa A. Andersch und H. W. Richter, Herausgeber von Der Ruf, als Sprecher der jungen Generation verstanden, obwohl sie am Kriegsende 31 bzw. 36 Jahre alt waren. 
Umsetzung der neuen, da verwandelten Zeit als Aufgabe in Aussicht gestellt wird. Das Jahr 1945 als Zäsur in diesem strengen Sinne zu nominieren, heißt somit, Kontinuität zwischen jetzt und damals zu bestreiten, die Stränge zwischen der Nachkriegszeit, Kriegszeit und der Zwischenkriegszeit ungültig oder zumindest unsichtbar zu machen.

Am anderen Pol dieses Schemas findet man all diejenigen vor, die mit einer solchen Zäsur-Auffassung des Jahres 1945 nichts am Hut hatten. Das Fragezeichen im Titel 1945 als Zäsur? stellt somit die Alleingültigkeit des Zäsur-Charakters von 1945 in Frage, indem es signalisiert, trotz des unumstrittenen realpolitischen Endes einer unheilvollen historischen Epoche, die in der bedingungslosen Kapitulation Deutschlands ihren Ausdruck und in den auf denazifizierende Umerziehung der Deutschen hin ausgerichteten, streng regulierenden Bedingungen der jeweiligen Besatzungszonen ihre Konsequenzen gefunden hat, mochte das Jahr 1945 für den intellektuellen, also auch den literarischen Betrieb eben nicht nur eine Zäsur dargestellt haben. Sosehr sich im besetzten Deutschland der politisch-kulturelle Rahmen komplett verändert hat, dessen reglementierenden Maßnahmen man sich von nun an anpassen musste, die Produzenten sowie die Konsumenten der Literatur konnten sich einfach mitnichten über Nacht geändert oder ausgetauscht haben, ${ }^{3}$ ja im Allgemeinen wird gar dem bundesrepublikanischen Literaturbetrieb attestiert, er habe sich in seinen Institutionen und Praktiken erst in den späten 1960er Jahren geändert (modernisiert), da er „noch mehr als ein Jahrzehnt nach dem Kriegsende seitab westlicher Entwicklungen vor sich hin gelebt ${ }^{\text {“4 }}$ habe. In allen produktiven Segmenten des kulturellen Lebens galt es nach 1945 somit, die an sich gegebene Kontinuität in den verwandelten Rahmenbedingungen in ein Selbstprofil umzuwandeln, das angesichts der Vergangenheit glaubwürdig (zumindest in den Augen der Kommissare der Besatzungsmächte) und im Hinblick auf Zukunft strategisch zu wählen war, damit der jeweilige Akteur im Nachkriegsfeld nachhaltig Fuß fassen konnte. Was in Bezug auf die Zäsurfrage heißen mag: Nach Zäsur des Jahres 1945 zu fragen, dürfte nur Sinn haben, wenn man statt pauschalisierenden Urteilen eher bei einzelnen Fällen eben die Strategien in den Blick nähme, mit deren Hilfe man die Spannung zwischen dem für den kulturellen Betrieb lebensnotwendig Langfristigen, Dauernden und Hergebrachten, den neuen kulturpolitischen Maßnahmen der Alliierten sowie den dem kulturellen Feld inhärenten Distinktionskämpfen auszubalancieren suchte. ${ }^{5}$ Der Neubeginn in der Form der strengen Zäsur wird dadurch vielmehr zu einem der Inszenierungseffekte, um

3 Dazu pointiert E. Schütz: „Nach 1945 wollten sie nun allesamt als Opfer gelten, neben den real Verfolgten auch die Verfolger, Opportunisten oder Lavierenden. Und sie schrieben weiter, ihre alten Bücher wurden wiederaufgelegt, sie taten sich in Akademien zusammen und verhalfen sich wechselseitig zu Preisen. Zwar bekämpften und verachteten sie teilweise einander, allermeist aber wahrten sie Stillschweigen übereinander, solange niemand daran rührte." Schütz, Erhard (2019): Mediendiktatur Nationalsozialismus. Heidelberg: Winter, S. 82.

4 Doering-Manteuffel, Anselm (1993): Die Kultur der 50er Jahre im Spannungsfeld von „Wiederaufbau“ und „Modernisierung“. In: Schildt, Axel - Sywottek, Arnold (hrsg.): Modernisierung im Wiederaufbau. Die westdeutsche Gesellschaft der 50er Jahre. Bonn: Dietz, S. 536.

5 Sehr anregend hier etwa der Blick auf die Verlagslandschaft vor und nach 1945 bei David Oels, der im Falle des Rowohlt Verlages die Strategie für die unmittelbare Nachkriegszeit als eine Mischung aus dem propagierten und massiv kommunizierten Neuanfangsgestus bei gleichzeitigem Fortwirken des Hergebrachten, das nicht expliziert worden, aber implizit bestimmend geblieben sei. Vgl. Oels, David (2013): Rowohlts Rotationsroutine. 
sich nach 1945 gegen die wohl dominierende und als unheilvoll empfundene Wirkung der Kontinuitäten behaupten zu können. Genauso sind aber innerhalb des erwähnten Spannungsfeldes andere Profilierungen gegenüber 1945 denkbar gewesen, bei denen man sich etwa dem Kontinuitätsbewusstsein nicht derart massiv entgegensetzt und die Vergangenheit, inklusive der eigenen biographischen der Jahre 1933-45, nicht primär via Zäsurgestus distanziert hatte.

Dazu kommt noch: Sozial-, kultur- und ideengeschichtliche Studien der letzten Jahrzehnte reden bezüglich der frühen Bundesrepublik von einer gewisser ideengeschichtlichen Trägheit, die sich etwa darin manifestiert, dass nach 1945 eine recht dynamische ökonomisch-technische Modernisierung von nicht weniger machtvollen Rückbesinnungsversuchen auf „den kulturellen und geistigen Horizont der 1920er Jahre "6 begleitet gewesen sei; die ideengeschichtlichen Prozesse mit ihren sehr langen Rhythmen gerieten nach 1945 offenbar in Spannung zu allen Bereichen, in denen man schneller umschalten konnte und musste. Daher einer der Impulse für dieses Themenheft, diesen längeren Rhythmen innerhalb der oben angedeuteten Spannungskonstellation nachzugehen. Wenn etwa F. Kießling schreibt, „[d]ie Ideengeschichte der alten Bundesrepublik ist vielfach, und deutlich stärker, als sie es in ihrem Selbstbild lange wahrhaben wollte, mit der ersten Jahrhunderthälfte verbunden." ${ }^{\text {"7 }}$, dann liegt es nahe, die hier angesprochene Diskrepanz zwischen dem langsamen Tempo der ideengeschichtlichen Transformationen und dem Bild, das mittels Zäsuren die auf größere Mobilität hin ausgerichtete Bundesrepublik von sich gezeichnet hatte, zugleich mit selbstpräsentierenden Praktiken von deren Akteuren zu verschränken. So unterschiedlich diese Praktiken auch gewesen sein mögen, sie mitzureflektieren scheint nützlich zu sein, egal, ob man die Zäsur 1945 offensiv herauszustellen, oder schon eine programmatisch bewahrende Einstellung zu den kurz oder lange vor 1945 etablierten intellektuellen, politischen und künstlerischen Traditionen an den Tag zu legen pflegte, denn: Problematisch war der (Rück)Blick vor das Jahr 1945 für alle, die sich nach 1945 im intellektuellen Nachkriegsfeld zu behaupten suchten, ob sie sich schon von den Jahren 1933-45 explizit distanziert und über sie hinweggesehen haben, um von Anfang an beginnen zu können, oder ob sie diese Jahre vielmehr ,nur beschwiegen haben und stattdessen an die Moderne der 1910er und 1920er Jahre anschließen wollten, um, als ,innere Emigranten', von der einen oder anderen Konzession ans NS-Regime nicht erzählen zu müssen, ohne die ein literarisch aktives Überleben der Totalität in Deutschland kaum möglich gewesen war.

Daraus ergaben sich die spannungsbeladenen Profilierungsbemühungen aller Akteure nach 1945. Die für die intellektuelle Landschaft nach 1945 prägende Polarisierung geht zum großen Teil auf eben diese Profilierungsaktivitäten zurück: Jüngere versus Äl-

\footnotetext{
Markterfolge und Modernisierung eines Buchverlags vom Ende der Weimarer Republik bis in die fünfziger Jahre. Essen: Klartext, S. 16.

6 Doering-Manteuffel [Anm. 5], S. 533.

7 Kießling, Friedrich (2011): „Gesprächsdemokraten“ - Walter Dirks“ und Eugen Kogons Demokratie- und Pluralismusbegründungen in der frühen Bundesrepublik. In: Gallus, Alexander - Schildt, Axel (hrsg.): Rückblickend in die Zukunft. Politische Öffentlichkeit und intellektuelle Positionen in Deutschland um 1950 und um 1930. Göttingen: Wallstein, S. 402.
} 
tere; die 1933-45 in Deutschland Verbliebenen (dem innerlich sehr heterogenen Feld der ,inneren Emigration‘ zuzuordnen) versus die Emigranten; die sich vor (zum Teil auch während des Krieges) Etablierenden versus diejenigen, denen es erst nach 1945 glücken sollte. Zwischen diesen Fronten entfachten sich in den ersten Nachkriegsjahren manche realpolitischen, symbolischen oder semantischen Kämpfe etwa darum, wer nach 1945 das Erbe des besseren, da vom Nationalsozialismus nicht korrumpierten Deutschlands wird repräsentieren dürfen oder welches Masternarrativ man der Erzählung über die intellektuelle und literarische Geschichte der Bundesrepublik zugrunde legen wird. Eines von diesen Narrativen, im Rückblick gesehen wohl das stärkste, lässt sich bereits aus den programmatischen Texten einiger wichtiger literarischer Zeitschriften herauslesen. Da Zeitschriften im Allgemeinen als Kristallisationspunkte und Verständigungsforen zu betrachten sind, deren Analyse Aufschluss über die zentripetalen sowie zentrifugalen Kräfte bestimmter intellektueller Gruppen geben kann, seien innerhalb der Einleitung drei Zeitschriften (Die Wandlung, Frankfurter Hefte, Der Ruf) kurz und - danach - eine (Merkur mit besonderem Blick auf J. Moras als einen deren Herausgeber) ausführlicher auf ihre profilierende Programmatik hin vorgestellt.

Im Geleitwort von Die Wandlung, gegründet bereits 1945, formulierte K. Jaspers im Auftrag des Herausgeberkreises (D. Sternberger sowie W. Krauss und A. Weber) die Zielsetzung durchaus im Sinne der Zäsur, sosehr alle verantwortlich Zeichnenden bereits seit der Zwischenkriegszeit ins intellektuelle Leben involviert waren. Da man alles verloren zu haben glaubte (Staat, Wirtschaft, Normen, Würde, Selbstbewusstsein) bis auf das Leben, habe es nun gegolten, sich aus dem Nichts heraus aufzuraffen, um von „vorn“8 anzufangen. Der Sicherheiten der Grundlagen beraubt, allerdings in den zwölf Jahren „innerlich und äußerlich verwandelt“9 ${ }^{\star 9}$ sah man sich nun mitten im Prozess weiterer Verwandlung begriffen, dessen Ergebnisse zwar nicht genau festlegbar, doch weitgehend absehbar gewesen seien: Einerseits vom nationalsozialistischen Joch befreit, andererseits dem „Willen der Besatzungsmächte“ ${ }^{10}$ ausgesetzt, hätte man sich auf einen Spielraum zu beschränken, dessen Potenzial allerdings voll auszuschöpfen war, um die Wandlung zu einer öffentlichen und möglichst freien Angelegenheit zu machen. Symptomatisch hier die Programm- und Zielabstinenz, sowie eine klare Absage an jedes, durch den Nationalsozialismus restlos kompromittierte Propheten- oder Führertum: „über den Dingen zu stehen, einen Plan des Ganzen zu entwerfen“11, sei eine unerlaubte Anmaßung gewesen, die an totalitäre letzte Standpunkte erinnere. Kurzum: Man könne allenfalls den Boden bereiten, auf dem - „in Meditation und Diskussion“, ${ }^{12}$ in gegenseitiger Toleranz und Solidarität die womöglich herrschaftsfreie Kommunikation der verantwortlichen, einander vertrauenden Individuen geübt werden kann.

8 Zit. nach: Pross, Harry (1963): Literatur und Politik. Geschichte und Programme literarisch-politischer Zeitschriften im deutschen Sprachgebiet seit 1870. Olten: Walter, S. 319.

9 Ibidem, S. 318.

10 Ibidem.

11 Ibidem, S. 318.

12 Ibidem, S. 319. 
Die linkskatholischen Frankfurter Hefte, gegründet 1946 von W. Dirks und E. Kogon, begaben sich im abenteuerlichen Augenblick des neuen Anfangs auf die Suche nach ihren potenziellen Lesern, einer unbekannten Größe, von der man nicht mehr wusste, als dass sie zu Partnern, Teilhabern und Freunden dieser Zeitschrift habe werden können. Zugemutet wurde dieser Leserschaft, die nun zu mündigen und nachdenklichen Christen erzogen werden sollen, dass sie selbst den harten und unbequemen Wahrheiten aufgeschlossen zu bleiben habe. Somit wurde einer mutigen Selbstaufklärung der Nation das Wort geredet, die sich auch ausgiebig der entsprechenden Lichtmetaphorik bediente. Die vergangenen Jahre wurden als ein abgründiges Dunkel, „das Undurchsichtige und das Rätselhafte“13 bezeichnet, das sich nun zu „lichten“ und „klären“ habe, damit das Nebelwort der Phrasen einer guten Sicht, einem „präzise funktionierenden Verstand“ ${ }^{\prime 14}$ mit dem lebendigen Herz, das „im Rhythmus der Zeit für die ewigen Ziele schlägt“"15, Platz machen könnte.

Am offensivsten dürfte sich des Zäsurgestus Der Ruf mit dem Untertitel Unabhängige Blätter der jungen Generation bedient haben, eine 1946 von A. Andersch und H. W. Richter gegründete Zeitschrift, die mit dem zwischen März 1945 und April 1946 in den Vereinigten Staaten herausgegebenen Der Ruf. Blätter für deutsche Kriegsgefangene eine veritable Vorgeschichte vorzuweisen hatte, und trotz des baldigen Verbots zum Grundstein der Gruppe 47 geworden ist. Von den Zeitschriften der ersten Stunde wird gerade diese wohl am nachdrücklichsten die Zäsur des Jahres 1945 inszeniert haben, indem sie den Neuanfang auf eine geradezu beschwörende Art gesetz ${ }^{16}$ und in einer recht eigenwilligen Interpretation zum Privileg eben der Kohorte der jungen deutschen Generation „zwischen 18 und 35“17 erklärt hat. Diese Generation zum womöglich ausschließlichen Träger „im Prozeß einer Weltwende“"18 zu erklären, war allerdings nicht naheliegend, musste zunächst rhetorisch geleistet werden. Wie dies geschah, ist einigen programmatischen Texten von Andersch und Richter zu entnehmen.

In Das junge Europa formt sein Gesicht blickt man auf die Nachkriegslandschaft zunächst von einer makroskopischen Einstellung aus („Ameisenberg Europa“, „Gewimmel der Millionen“, „neue Kräfte und Willenszentren“"19), die um mythologische Anspielungen („wie einst dem Haupt des Jupiters die Athene ${ }^{“ 20}$ ) angereichert wird, damit die nun zu privilegierende Jugend, in deren Namen man spricht, im Zeichen der Vitalität (jugendfrisch) und zugleich Reinheit (jungfräulich) erscheinen kann. Vital sei man, eigenwillig genug, da man die Zeit nicht „in der Stille von Studienzimmern“ vergeudet („dazu hat-

13 Ibidem, S. 324.

14 Ibidem.

15 Ibidem.

16 vgl. Veit, Walter (1981): Die Stunde Null im Spiegel einiger zeitgenössischer Zeitschriften. In: Hüppauf, Bernd (hrsg.): Die Mühen der Ebenen. Kontinuität und Wandel in der deutschen Literatur und Gesellschaft 1945-1949. Heidelberg: Winter, S. 222.

17 Pross [Anm. 9], S. 335.

18 Ibidem, S. 331.

19 Ibidem.

20 Ibidem. 
ten sie keine Zeit“" ${ }^{\text {21 }}$ ), sondern sinnvoll in der unmittelbaren Aktion, konkret im „bewaffneten Kampf um Europa“22 verbracht habe. Das positiv konnotierte Kriegserlebnis, mal als ein religiöses, in der Freiheit für oder Gott sich manifestierendes, ein anderes Mal einfacher als „Front- und Gefangenschaftserlebnis “23 formuliert, wird in der Darstellung von Andersch - erstens - massiv vom Hitlerdeutschen nationalsozialistischen Kriegsengagement getrennt, damit - zweitens - deren Träger ihre Führungsrolle für das deutsche Nachkriegsfeld innerhalb der europäischen Konstellation beanspruchen können. Darum nicht nur die sehr bedenklichen Bezeichnungen wie „Kampf um Europa“ für den Zweiten Weltkrieg, doch vor allem weitere antifaschistische Signale, die im Text zu finden sind: Andersch, der bekanntlich im Juni 1944 desertierte, reklamiert nun für seine Zeitschrift das Erbe der gesamten europäischen antifaschistischen Kräfte der „résistance“, deren sozialistischem Humanismus er sich anschließt. Eine Zäsur wird somit gesetzt, indem man sich von der Zeit bis 1945 die Verbündeten ausschließlich bei den Kräften des europäischen Widerstands sucht, um das Gesicht des jungen Europas nach 1945 als Bestandteil dieses zu einem Monolith zurechtgebogenen antifaschistischen Blocks gestalten zu können. Dass dieser Akt eine Selbstentlastung miteinschließt, wird Andersch nicht entgangen sein (,ein dünnes, sehr gewagtes Seil über einen Abgrund hinweg““24), doch er bemüht ihn dennoch, so fragwürdig auch seine Argumente sind. Das erste ist die sogenannte Haltung: Dieses junge Deutschland habe zwar (1933-45) für eine falsche Sache gestanden, doch es habe gestanden, habe also eine Haltung bewiesen. Das zweite Argument läuft auf eine Selbstexkulpation hinaus, die auf dem Recht gegründet ist, die Fehler eingesehen und dazugelernt zu haben: Den Brückenschlag zwischen „den alliierten Soldaten, den Männern des europäischen Widerstandes und den deutschen Frontsoldaten, zwischen den politischen Häftlingen und den ehemaligen Hitlerjungen "25 bemüht Andersch, indem er (selbstentlastend) die Verbrechen einer Minderheit zuschiebt und den ehemaligen Hitlerjungen das Recht auf Wandlung zuerkennt (,sie sind es schon längst nicht mehr!“26). Das dritte Argument schließlich spricht dieses Recht, sich dank Einsicht in eigene Fehler verwandeln zu können, allen anderen Generationen ab, denen entweder (den Älteren) ${ }^{27}$ die Verantwortung für Hitler angelastet wird, oder (den Jüngeren) diese Front- und Gefangenschaftserlebnisse streitig gemacht werden.

Begleitet wird diese Argumentationskette von etlichen Bedenken gegenüber der oktroyierten ,reeducation“ („Hat man sich einmal wirklich vorgestellt, wen man rückerziehen will? [...] Wenn Bildung nicht Belehrung bleibt, sondern zum tiefsten Erlebnis wird

\footnotetext{
21 Ibidem.

22 Ibidem.

23 Ibidem, S. 333.

24 Ibidem.

25 Ibidem.

26 Ibidem.

27 Die ältere deutsche Generation soll „in der Unverbindlichkeit ihres Toleranz-Begriffs, ihrem Zurückschrecken vor dem letzten Einsatz, dem Unhold seinen Gang zur Macht erlaubt“ haben. Ibidem.
} 
$\left.[\ldots]^{(28}\right)$, die sich allerdings überhaupt nicht auf die bewusst vollzogene Wandlung als eigene Leistung beziehen, die den Kräften der deutschen Kriegsgefangenschaft in den USA (denen eben Der Ruf entsprang) eingeräumt wird. Genauso ausgenommen werden auch die versprengten Gruppen der Geflüchteten, die nach ihrer Rückkehr (wieder ist die Rede ,nur‘ von Amerika) den deutschen Beitrag zum Nachkriegseuropa mitzuleisten hätten.

Die Frontstellung, aus der heraus man sich in Ruf im intellektuellen Nachkriegsfeld profilieren wollte, ist frappierend einfach: Unter der Schirmherrschaft des europäischen antifaschistischen Widerstands und mit Unterstützung der nach und nach heimkehrenden deutschen Emigranten erklärt man die diesen progressiven Kräften angeglichene junge Generation der jeder Verantwortung für Hitler freigesprochenen deutschen Soldaten, die nach einer Lektion der Demokratie in den amerikanischen Gefangenenlagern keiner weiteren frontalen Umerziehung und Denazifizierung mehr zu bedürfen glauben, für die einzige glaubwürdige Nachkriegsstimme Deutschlands. Das hier skizzierte Bild änderte sich in anderen programmatischen Texten von Andersch oder Richter nur minimal, allenfalls wurden die selbstentlastenden Momente ${ }^{29}$ sowie die abhebende Profilierung gegenüber anderen Generationen und Gruppen gesteigert oder - in schwächeren Momenten - um ideologische Ressentiments gegenüber der Emigration erweitert. Das wohl schillerndste Beispiel einer intergenerationellen Selbstentlastung findet sich in Anderschs Notwendige Aussage zum Nürnberger Prozeß (15.8.1946), wo man sich ausdrücklich dagegen verwahrt, die „erstaunlichen Waffentaten junger Deutscher in diesem Kriege“ ${ }^{\text {“30 }}$ mit den zur Zeit in Nürnberg verhandelten Verbrechen „etwas älterer Deutscher“31 etwa von Dachau und Buchenwald in jedweden Zusammenhang zu stellen: den Kämpfern von Stalingrad oder Cassino sei keine Schuld an den Verbrechen zuzuschreiben, ihnen sei von deren Gegnern vielmehr ,jede Achtung entgegengebracht" ${ }^{* 32}$ worden. Und im Text Literatur im Interregnum (15.3.47), von dem spätere Ausgaben Richters wenig wissen wollten, ist die Zäsur von 1945 nicht mehr nur an die Kriegsverbrechen gekoppelt, von denen man sich via Zäsur lossagt, hier werden ganze Epochen inklusive deren Genera-

\footnotetext{
28 Ibidem, S. 334.

29 Etwa in Warum schweigt die junge Generation H. W. Richters (2.9.1946): Der jungen Generation bleibt in dieser Schilderung nichts anderes übrig als zu schweigen, demotiviert durch die Generation der Alten, die ,gleichsam in eine Wolke von bußfertigem Weiherauch gehüllt, in die beruhigenden Schatten der Vergangenheit flieht" und den öffentlichen Raum wieder dominiert, indem sie der jungen Deutschen, deren sich Der Ruf annimmt ,jede sittliche und geistige Fähigkeit mit professoraler Selbstverständlichkeit“ abspreche, während sie ihr nur den moralischen, geistigen und sittlichen Trümmerhaufen zurückgelassen gehabt habe. Die junge Generation wird hier grandios entlastet (sie sei gestern gezwungen worden, die Handgranate zu entsichern, sie sei in diese Welt hineingewachsen, in der sie sich kämpfend zu bewähren suchte) und der Verantwortung schlechthin (NS wird als ein vulkanischer politischer Ausbruch stilisiert) freigesprochen (sie habe die Niederlage erlitten, und es komme heute überhaupt nicht darauf an, ob sie dieses Regime gehasst oder bewundert gehabt habe). Vgl. Richter, Hans Werner (1976): Warum schweigt die junge Generation? [Der Ruf, Nr. 2 / 2. September 1946]. In: Neunzig, Hans A. (hrsg.): Der Ruf. Unabhängige Blätter für die junge Generation. München: Nymphenburg, S. 60-65.

30 Ibidem, S. 26.

31 Ibidem.

32 Ibidem.
} 
tionen, Ideenträger und literarischer Repräsentanz zum Tode verurteilt, um den neuen Platz zu machen.

Nach dem Verbot von Ruf wurden einige seiner programmatischen Punkte in leicht modifizierter und politisch korrekterer Form der Gruppe 47 zugrunde gelegt; für unsere Fragestellung sei insbesondere auf das wohl zentrale Masternarrativ verwiesen, in dem die interessenbedingte Sicht insbesondere der Gruppe 47 zum prägenden und im Laufe der Jahre zunehmend kanonisierten ${ }^{33}$ Blick auf die gesamte Nachkriegszeit wurde. In einer vereinfachten Form setzt dieses Narrativ eben an dem hoffnungsüberladenen Aufbruch der ,Stunde Null' an, deren Erwartungen allerdings bald aus der Sicht derjenigen, die 1945 als Zäsur inszeniert hatten, enttäuscht, da durch die dunklen ,bleiernen“ 1950er Jahre der Restauration und deren moralisch unheilvollen Repräsentanten zunichtegemacht wurden: Die Bundesrepublik habe die einzigartige Chance zur geistigen Erneuerung vertan, statt der Säuberung und Revolutionierung des öffentlichen Lebens seien alle „Krankheitskeime“34 virulent geblieben, durch die Kollektivschuldthese seien paradoxerweise die NS-Kader entlastet worden, die nun, statt der jungen, unbelastet geglaubten Intellektuellen ihre alten Kontakte von ehedem reaktiviert und in der jungen Bundesrepublik fatalerweise wiederum die Macht erobert hätten. Anstatt Revolution eines sozialistischen Humanismus im Namen eines neuen Deutschlands sei es zu einem moralischen Bankrott gekommen, statt eines Anfangs mit gerechten Chancen würden in der Konstellation des Kalten Krieges immer wieder diejenigen privilegiert, ${ }^{35}$ die eben erst geschlagen worden wären.

Diese Sicht auf das erste Nachkriegsjahrzehnt, in dem die unheilvollen Tendenzen der Restauration kulminiert haben sollen, leuchtet aus dem Rückblick ein, wäre problemlos mit etlichen konkreten Beispielen zu belegen, in ihrer einseitigen Pauschalität ist sie allerdings kaum zu halten, darum in manchen Punkten zumindest auf ihren interessenbedingten Ursprung hin zu befragen. Dass dies seit geraumer Zeit geschieht, zeigen nicht nur einige selbstrevidierende Bekenntnisse der zentralen Protagonisten der Gruppe 47,36 sondern auch etliche Studien, die das erste Nachkriegsjahrzent anders strukturieren,

33 Neben Ruf wurde der Begriff der Restauration im Hinblick auf die 1950er insbesondere in Frankfurter Heften stark gemacht. Zur massiven Kanonisierung des Begriffs in den deutschsprachigen Literaturgeschichten nach 1945 vgl. Kiesel, Helmuth (1997): Die Restaurationsthese als Problem für die Literaturgeschichtsschreibung. In: Erhart, Walter - Niefanger, Dirk (hrsg.): Zwei Wendezeiten. Blicke auf die deutsche Literatur 1945 und 1989. Tübingen: Max Niemeyer, S. 13-46.

34 Richter, Hans Werner (1962): Zwischen Freiheit und Quarantäne. In: ders. (hrsg.): Bestandsaufnahme. Eine deutsche Bilanz 1962. München: Kurt Desch, S. 13.

35 Dieser Gestus der zu Unrecht Zukurzgekommenen prägte bereits die Texte im Ruf, doch auch die späteren Texte Richters wurden von ihm dominiert, etwa die in dem von ihm herausgegebenen Band mit dem Titel Bestandsaufnahme. Eine deutsche Bilanz 1962, in dem in 36 Beiträgen die ersten 15 Nachkriegsjahre charakterisiert werden. Die Bilanz, zumindest bezüglich der literarischen Entwicklung, kommt um den Begriff der Restauration nicht herum, auf den es in den 1950er Jahren aus der Sicht der um den Erfolg Gebrachten hinausläuft.

36 Als Beispiel hierzu der seine eigenen Irrtümer kaum verheimlichende H. M. Enzensberger, der bereits Ende der 1980er einräumte: „Die Rede von der Restauration, ein in den fünfziger Jahren beliebter Topos, beruhte, wie wir heute wissen, auf einer Augentäuschung“. Enzensberger, Hans Magnus (1988): Mittelmaß und Wahn. Frankfurt am Main: Suhrkamp, S. 251. 
indem sie neue Akzente in Betracht ziehen. Nach manchen empirischen und kritischen Betrachtungen wurde - der Reihe nach - zunächst der absolute Zäsurcharakter der ,Stunde Null' deutlich abgeschwächt, wenn nicht ganz abgelehnt: Dies geschah durch Hinweise darauf, dass 1945 vielmehr „Stunde äußersten physischen und ideologischen Elends, die Stunde der Unfähigkeit zu kritischem Denken, die Stunde der Anfälligkeit für die geringsten Tröstungen“"37 gewesen sei, oder durch Entdeckungen mancher unerwünschter NS-Relikte (etwa auf sprachlicher Ebene ${ }^{38}$ ) in zentralen Texten der in ihnen proklamierten Stunde Null. Folgerichtig ergab die Abschwächung des Zäsurcharakters von 1945 eine Korrektur der 1950er Jahre, die trotz mancher restaurativer Tendenzen aus dem manichäischen Bild herausgenommen, und vielmehr einen ambivalenten, janusköpfigen Charakter gewonnen haben. Schwarz-weiße und monolithische Bilder von der Restauration (so wie von der Stunde Null) weichen nun allmählich komplexeren, die von einer Koexistenz der Traditions- und Modernitätsbegriffe der 1950er Jahre ausgehen, und etwa für die bundesrepublikanische Kulturgeschichte eine produktive Spannung „zwischen einsetzender Modernisierung und ideologisch konzeptionellem Rückgriff auf mehr oder weniger traditionelle Bestände“39 konstatieren. Diese Gleichzeitigkeit „von Bereitschaft zum Aufbruch in eine neue westliche Moderne [...] und dem Festhalten an überkommenen Deutungsmustern“ ${ }^{40}$, die laut A. Schildt spätestens für die zweite Hälfte der 1950er Jahre prägend gewesen sein soll, ${ }^{41}$ sowie die biographischen Kontinuitäten, deren Radius oft weit bis in die Zwischenkriegszeit reichen mag, liegen als Herausforderung vielen in unserem Band publizierten Beiträgen zugrunde, werden darin doch, um nur ein Beispiel zu nehmen, etwa mit der rowohlts deutschen enzyklopädie Projekte aus der Mitte der 1950er Jahre analysiert, deren Konzeption und Praxis genau in dem komplexen Spannungsfeld zwischen alt und neu, traditionell und modern, national und international zu verorten sind. Zum Teil in freier Weiterentwicklung einiger doch schon älterer Impulse von A. Schildt ${ }^{42}$ bemühen sich die diesem Komplex gewidmeten Beiträge darum, anhand ausgewählter Segmente den Blick auf das erste Nachkriegsjahrzehnt in Deutschland, Österreich und der Schweiz von den Alternativen 1945 als Zäsur oder

37 Vormweg, Heinrich (1981): Deutsche Literatur 1945-1960: Keine Stunde Null. In: Durzak, Manfred (hrsg.): Deutsche Gegenwartsliteratur. Ausgangspositionen und aktuelle Entwicklungen. Stuttgart: Metzler, S. 17.

38 Vgl. Widmer, Urs (1966): 1945 oder die „neue Sprache“. Studien zur Prosa der „Jungen Generation“. Düsseldorf: Schwann, S. 198-199.

39 Fischer, Ludwig (1993): Zur Sozialgeschichte der westdeutschen Literatur. In: Schildt, Axel - Sywottek, Arnold (hrsg.): Modernisierung im Wiederaufbau. Die westdeutsche Gesellschaft der 50er Jahre. Bonn: Dietz, S. 559.

40 Schildt, Axel (1999): Zwischen Abendland und Amerika. Studien zur westdeutschen Ideenlandschaft der 50er Jahre. München: Oldenbourg, S. 197.

41 vgl. Ibidem.

42 A. Schildt erschloss Ende der 1990er Jahre einige neue Themen und Bereiche, die unser Bild von den monolithisch restaurativen 1950er Jahren korrigieren könnten (Popularisierung der Wissenschaft durch Taschenbuchreihen oder wissenschaftlich kulturelle Reihen anderer Verlage wie Fischer, List, Piper, Ullstein, Vortragstätigkeit verschiedener Clubs und Foren, Zeitschriftenpublizistik). vgl. Ibidem, S. 8-13. In diese Richtung begab sich: Kießling, Friedrich (2012): Die undeutschen Deutschen. Eine ideengeschichtliche Archäologie der alten Bundesrepublik 1945-1972. München - Wien - Zürich: Schöningh. 
1945 als Kontinuität freizumachen und hin zum darin enthaltenen spannungsvollen und ambivalenten Wechselspiel von Neuanfang und Kontinuität zu öffnen. Trotz ihrer unterschiedlichen Ansätze nehmen sich die Beiträge vor, die ideologisch bedingten und generationell verfestigten Schemata eben auf deren Überlappungen und Kreuzschnitte hin zu untersuchen. Dies scheint übrigens im Einklang mit der Forschung der letzten Jahre zu sein, die nun von den notorisch untersuchten Jahren 1918, 1945, 1968 zunehmend absieht, und stattdessen die Vielzahl der ideengeschichtlichen Kontinuitäten zwischen der Zeit um 1930 und dem ersten Nachkriegsjahrzehnt ${ }^{43}$ unter die Lupe nimmt sowie der Dynamik ${ }^{44}$ zwischen den als Konstrukt verstandenen Generationen, Halbgenerationen oder Zwischengenerationen nachgeht (hier insbesondere der lange unterbelichteten Generation der zwischen 1900 und 1910 Geborenen, die im ersten Nachkriegsjahrzehnt relativ schnell ihre Rolle einer zwischen den Alten und sehr Jungen vermittelnden Zwischengeneration zu institutionalisieren vermochte). Geleitet dürften diese Studien dabei sowohl durch viele Einzelbeobachtungen als auch durch die grundlegende Annahme von A. Schildt gewesen sein, die greifbaren Auswirkungen all der gruppenbiographischen Kontinuitäten, die ja „durch weit zurückreichende Netzwerke und nützliche Freundschaften in kirchlichen, konservativen oder liberalen Milieus, auch in Universitätsorten" ${ }^{45}$ befördert wurden, seien erst mit gewisser Verspätung, also etwa um 1950 transparent geworden, nachdem sich der „Nebel der Nachkriegsunübersichtlichkeit weitgehend gelichtet" ${ }^{* 6}$ hätte. Dass zu einem differenzierten Bild der ersten Nachkriegsjahrzehnte auch manch ein Blick direkt in den kaum durchsichtigen Nebel beitragen könnte, unter dessen Mantel sich die sich noch nicht manifestierenden Wieder- und Neubegegnungen abgespielt haben werden, steht freilich außer Frage.

\section{Merkur. Deutsche Zeitschrift für europäisches Denken}

Anschließend an diese einführenden Bemerkungen soll Merkur. Deutsche Zeitschrift für europäisches Denken auf seine Programmatik hin untersucht und als eine Zeitschrift vorgestellt werden, deren programmatische Aussagen wie auch Biographien des engen Herausgeberteams, das Hans Paeschke und Joachim Moras bildeten, sowie der Autoren der ersten Jahrgänge den Zäsurcharakter des Jahres 1945 radikal in Frage stellen. ${ }^{47}$ An

43 Gallus, Alexander - Schildt, Axel (2011): Rückblickend in die Zukunft. Politische Öffentlichkeit und intellektuelle Positionen in Deutschland um 1950 und um 1930. Göttingen: Wallstein. S. 20ff.

44 Laut M. Payk gehe die Generation nicht in der rechnerischen Zugehörigkeit zu bestimmten Geburtsjahrgängen auf, sondern sei „eine diskursiv konstruierte Kategorie [...], deren identitätsstiftendes Potential in dynamischen Aushandlungs- und Beeinflussungsprozessen hergestellt wird“. Payk, Marcus (2008): Der Geist der Demokratie. Intellektuelle Orientierungsversuche im Feuilleton der frühen Bundesrepublik: Karl Korn und Peter de Mendelssohn. Oldenbourg: De Gruyter, S. 366.

45 Gallus, Alexander - Schildt, Axel [Anm. 44], S. 22.

46 Ibidem.

47 Zum Teil basieren folgende Ausführungen auf Ergebnissen, die bereits andernorts publiziert werden. Vgl. Urválek, Aleš (2020): „Diagonallinie der goldenen Mitte“ zwischen Eigenem und Fremdem. Konstellationen der frühnachkriegsdeutschen Internationalisierung am Beispiel von Merkur. Deutsche Zeitschrift für europäisches 
Merkur, dieser bisher verhältnismäßig wenig erforschten Zeitschrift, der meist bescheinigt wurde, im ästhetischen Sinne eine Zufluchtsstätte des europäischen Konservativismus darzustellen, ist daher im Folgenden insbesondere die Art relevant, wie man in dieser Zeitschrift, die 1947 an den Start ging, die Spannung zwischen Kontinuität und Diskontinuität zu der Zwischenkriegs- sowie der Kriegszeit auszubalancieren suchte. Merkur gab sich von Anfang an im Sinne der damals üblichen Programmabstinenz möglichst unideologisch, so sehr man auch hier nicht umhinkonnte, das bedenkliche Argument der Haltung ins Spiel zu bringen. Mehrstimmig, dialogisch und dezidiert offen strebte man an, alle erdenklichen Positionen dem freien Spiel der Argumente zu überantworten, als würde die Zeitschrift ihre Rolle darin erblicken - eine Art gedruckter Salon, Forum und Rundschau in einem -, Brücken zu bauen. Die proklamierte Ambition von Merkur war, „die verschiedenen geistigen Positionen ebenso wie die Generationen und die Nationen in die Begegnung und, wenn möglich, ins Gespräch zu führen “. ${ }^{48} \mathrm{Im}$ Gegensatz etwa zu Ruf war die Brücke großzügiger angedacht, vermittelt wurde somit nicht nur zwischen den deutschen und europäischen Soldaten, die von ihrem „Kampf um Europa“ heimgekehrt, in dessen Verlauf sie entweder bei den Widerstandskräften oder (zumindest) in den Gefangenschafslagern gelandet sind, sondern, in- und extensiver, zwischen den einzelnen Generationen, Zeiten, Nationen sowie den einzelnen wissenschaftlichen Disziplinen. Die Brückenmetapher war nicht die einzige, der man sich in Merkur bediente, positioniert hatte man sich auch an Schnittstellen zwischen links und rechts, zwischen Tradition und Experiment, ja an den Schnittpunkten zwischen Naturund Geisteswissenschaft, „Moral und Technik, Metaphysik und Psychologie, zeitlosem und zeitgebundenem Geist“. ${ }^{49}$ In den Fußstapfen von Wieland und Goethe glaubte man, die „Diagonallinie der goldenen Mitte“50 zu hüten. So sehr manche der kunstreichen Wendungen eher wie begriffliches Jonglieren aussahen, ${ }^{51}$ sie waren in der Tat durch das Bestreben motiviert, in der äußerst schwierigen Nachkriegskonstellation einen glaubwürdigen deutschen Anschluss an die besten europäischen Aufklärungstraditionen zu finden. In einer Zeit, die zu offensiver Programmatik und schnellen Lösungen tendierte, legten die Merkurianer eine bedacht aufklärerische Strategie an den Tag, insofern sie naheliegenden Scheinlösungen, falschen und uneigentlichen Gegensätzen und gleisnerischen Antithesen aus dem Weg zu gehen suchten. Oder noch anders: Jedes Denken war zu meiden, das nur reflexartig re-agiert, ${ }^{52}$ sei es indem es Gegensätze aufbaut, wo es sie

Denken. In: Hehl, Michael Peter - Tommek, Heribert (hrsg.): Transnationale Akzente. Zur vermittelnden Funktion von Literatur- und Kulturzeitschriften im Europa des 20. Jahrhunderts. Berlin: Peter Lang, S. 79-100. Dort siehe auch die bibliographischen Hinweise, hier sei aus Platzgründen nur auf zwei englischsprachige Titel verwiesen: Bailey, Christian (2013): Between Yesterday and Tomorrow: German Visions of Europe, $1926-1950$. New York - Oxford: Berghahn; Parker, Stephen - Davies, Peter - Philpotts, Matthew (2004): The Modern Restoration. Re-thinking German Literary History 1930-1960. Berlin - New York: De Gruyter.

48 Moras, Joachim - Paeschke, Hans (1954): Nummer 100. In: Merkur 100, S. 504.

49 Ibidem.

50 Paeschke, Hans (1978): Kann keine Trauer sein. In: Merkur 367, S. 1170.

51 So etwa die Charakteristik, Merkur pflege „fruchtbare Zerrissenheit“. Kießling, Friedrich (2014): Fruchtbare Zerrissenheit. Der Merkur in der frühen Bundesrepublik. In: Zeitschrift für Ideengeschichte 1, S. 96.

52 vgl. Ibidem. 
nicht gibt, oder sie, da wo sie auszuhalten sind, dialektisch auflöst.

Insofern könnte der Kontrast zu Ruf gar nicht größer gewesen sein. Statt suggestiven Setzungen der Zäsur von 1945 wägte man im Merkur ab, inwiefern den ,Null-Punkten“ oder ,Stunde Null'-Gesten überhaupt zu vertrauen ist. Ultimatives Denken, das „vom Nullpunkt aus dachte“" 53 um via Zäsur die absolute Erneuerung sowie das Ende des zu Erneuernden zu proklamieren, wich in Merkur einer komplexeren, wenn auch nicht unumstrittenen Profilierung: Auf deren erste bescheidene Komponente - als ein deutscher Schriftsteller sei man mit seinem „Tun und Lassen unlöslich in die Verantwortung des gemeinsamen Dramas verstrickt “54 - folgte allerdings der selbstbewusste Nachsatz, man habe dennoch das Recht, sich am geistigen Gespräch in Europa zu beteiligen. An der Erklärung Paeschkes, der 1948 zu Protokoll gab, dass „der deutsche Geist“ ohne den der anderen Nationen nie er selbst geworden wäre, genauso wie der europäische Geist ohne den deutschen nicht europäisch hätte werden können, ${ }^{55}$ irritiert wohl nicht unbedingt der souveräne Gestus, zwei Jahre nach dem Kriegsende eine Stimme im Orchester der europäischen Stimmen auch der deutschen vorzubehalten, vielmehr das Vokabular („der deutsche Geist“), das seinen nationalistischen Ursprung verrät und darum einer eingehenden ideengeschichtlichen Analyse der Wurzeln dieses Konzepts in der Zwischenkriegszeit und deren Transformation in der Nachkriegszeit bedarf, zu der bereits erste Ansätze vorliegen. ${ }^{56}$ Hier sei deshalb stattdessen darauf verwiesen, dass die Abneigung, sich vom Vorherigen um jeden Preis loszusprechen, um vermeintlich „vom Nullpunkt aus“ anzufangen, im Merkur sehr wohl auch die Einstellung zum Nationalen nach 1945 mitbestimmte. Und zwar auf eine Art, die dann doch zu denken geben dürfte, so problematisch ihre ideengeschichtlichen Wurzeln auch sein mögen. Statt in der Konstellation der ersten Nachkriegsjahre eilfertig nach naheliegenden (Reflex-) Lösungen zu suchen, wären sie doch schon vom antinationalen Spott oder nationalen Trotz diktiert gewesen, strebte man eine womöglich reflektierte Balance in der Mitte an. Man formulierte sie überwiegend im mahnenden Sinne als Aufforderung, in der Nachkriegskonstellation nicht noch einmal das Deutsche im nationalen Sinne als Wert an sich zu hypostasieren, um sich entweder nur dem Deutschen (der nationalen Sicht) oder aber nur dessen Negation (dem Anti- oder Internationalen) zu verschreiben. Wie schwer, wenn nicht unmöglich es war, die Balance konsequent zu halten, ist den nicht seltenen Versuchen anzumerken, an all das zu erinnern, was der „deutsche Geist“ in der Vergangenheit hervorgebracht hätte, und nun zu verdrängen im Begriffe sei, ${ }^{57}$ werden

53 Paeschke, Hans (1963): In Sachen Merkur. In: Merkur 179, S. 5.

54 Moras, Joachim - Paeschke, Hans (1948): An unsere Leser. In: Merkur 10, S. 482.

55 vgl. Ibidem.

56 Ansätze dazu insb. Bock, Hans Manfred (2001): Die fortgesetzte Modernisierung des Konservativismus. Merkur: Deutsche Zeitschrift für europäisches Denken 1947-1957. In: Grunewald, Michel - Bock, Hans Manfred (hrsg.): Der Europadiskurs in den deutschen Zeitschriften (1945-1955), Bern: Peter Lang, S. 149-185. Eine äußerst kritische Lektüre des Ansatzes von Curtius, die diesen in die unheilvolle Tradition des ,unpolitischen Denkens der Weimarer Republik einbettet, liefert: Bock, Hans Manfred (1990): Die Politik des ,Unpolitischen“. Zu Ernst Robert Curtius' Ort im politisch-intellektuellen Leben der Weimarer Republik. In: lendemains. Études comparées sur la France 59, S. 16-61.

57 vgl. Paeschke, Hans (1954): Der Januskopf. In: Moras, Joachim - Paeschke, Hans - Einsiedel, Wolfgang 
diese doch stets vom essenziellen Verständnis der Nation aus formuliert, das man aber zugleich hinter sich gelassen haben wollte.

Da man Extreme meiden und stattdessen eine Position der Mitte einnehmen wollte, kamen für Merkur weder nationalistische noch antinationalistische Konzepte in Frage, umso mehr fühlte man sich durch die in der Zwischenkriegszeit formulierten Ansätze angesprochen, die - vereinfacht gesagt - das Nationale durch das als das Europäische verstandene Über-, Trans- oder Supranationale erdeten, um der Sackgasse zwischen engem Nationalismus und uferlosem Antinationalismus zu entkommen. Und dieser Antinationalismus hieß in den 1920er Jahren Internationalismus, so dass auch alle Nachkriegsversuche, den so verstandenen Antinationalismus auf Distanz zu halten, dem nicht so leicht von der Hand zu weisenden Vorwurf ausgesetzt sind, in die proklamierte Suche nach der Mitte komme durch die Hintertür eine Substanz, im Falle von Merkur etwa ein deutschamalgamiertes Europa, dessen man sich, zumal in der dafür recht günstigen Konstellation des Kalten Kriegs, im impliziten Kampf gegen alle internationalistischen Bestrebungen bedienen konnte, seien diese schon als nicht-europäisch, nicht-abendländisch, kommunistisch, bolschewistisch oder sozialistisch identifiziert worden.

Diese an sich nicht unproblematische ideengeschichtliche Kontinuität mit ihren politischen Konsequenzen ist im Merkur in einen breiteren Rahmen einzubetten, in dem man nach 1945 im weitesten Sinne an derart europäisch denkende Kreise, Foren, Zeitschriften, Konzepte, Ideen, Denker, Literaten anzuschließen gedachte, sowie in einen engeren, da die Merkurianer dadurch zugleich das wiederaufgegriffen haben, worin und womit sie selbst, also biographisch- intellektuell, aufgewachsen waren. In ästhetisch-programmatischen Worten von E. R. Curtius formuliert, in dem die Herausgeber H. Paeschke und J. Moras eine zumindest für die ersten Jahre von Merkur unumstrittene geistige Autorität gefunden haben, die ihnen Texte, Ratschläge, Kritik aber auch wichtige Kontakte ${ }^{58}$ zur europäischen Geisteselite vermittelte: Die Aufgabe,

„das System geistiger Kultur wiederherzustellen [...] lässt sich nicht in vier oder fünf Jahren wieder reparieren [...] wird nur gelingen, wenn wir an die Welt vor 1933 wieder anknüpfen, also an eine Zeit, die fast in Vergessenheit geraten ist [...] Aber es gibt, so dürfen wir hinzufügen, eine Tradition europäischen Geistes. Wir müssen nur den Zugang zu ihr finden. Das ist wichtiger und wirksamer als alle Umerziehung und alle Experimente. “59

Unter der geistigen Obhut von E. R. Curtius sowie weiteren engeren Beratern und Mitarbeitern, die sich nicht selten aus dem Umkreis der einstigen Schüler und Kollegen

von (hrsg.): Deutscher Geist zwischen gestern und morgen. Stuttgart, DVA, S. 453. Moras, Joachim: (1954): Die Mitte Europas. In: Moras, Joachim - Paeschke, Hans - Einsiedel, Wolfgang von (hrsg.): Deutscher Geist zwischen gestern und morgen. Stuttgart: DVA.

58 Kritiker wie M. Rychner, bei dem Curtius nach dem Krieg gewohnt hat, oder E. Vietta, aber auch A. Gide, T. S. Eliot, J. Ortega y Gasset, M. de Unamuno, P. Valéry verdanken ihre Präsenz in Merkur der Vermittlung von E. R. Curtius. Das Gleiche gilt auch für Emerson, Du Bos, Perez de Ayala, Toynbee, Guillén, Goyen, Giraudoux, Jouhandeau.

59 Curtius, Ernst Robert (1949): Ortega y Gasset. In: Merkur 5, S. S. 418-419. Später abgedruckt in: ders. (1954): Kritische Essays zur europäischen Literatur. Bern, S. 268. 
von Curtius rekrutierten, ${ }^{60}$ setzte man in Merkur das Kontinuitätsprogramm um, das zunächst zögernd, später zunehmend, dennoch nie programmatisch auch das Zeitgenössische (also Nachkriegsdeutsche) aufzunehmen bereit war. Insbesondere in den ersten Jahrgängen hatte man diverse Stränge der europäischen Kultur- und Literaturtradition der Zwischenkriegszeit aufgefrischt, modernen Klassikern wie J. Joyce oder P. Valéry sowie Vertretern der damaligen traditionalistischen Moderne wie T. S. Eliot oder M. Jouhandeau räumte man im Merkur der späten 1940er Jahre mehr Platz ein als den ersten wichtigen Repräsentanten der deutschen Nachkriegsliteratur, die erst nach und nach mit I. Aichinger, W. Höllerer, H. Bender, H. M. Enzensberger, W. Jens, ab Ende der 1950er Jahre auch mit G. Grass oder M. Walser vertreten waren. Diese tendenziell doch spürbare Neigung, ans europäische Denken und Schreiben der 1920er und 1930er Jahre anzuschließen, um sich von hier aus im Nachkriegsspannungsfeld zu behaupten, hat im Merkur neben anderem auch, wie angedeutet, biographisch-intellektuelle Wurzeln. Der engere Kreis der Merkur-Mitarbeiter, zu dem in den Anfangsjahren H. Paeschke, J. Moras, W. von Einsiedel und E. R. Curtius zählten, war, etwa anders als im Falle der Ruf-Herausgeber, bereits in der Zwischenkriegszeit netzwerkartig verbunden, insofern man sich entweder persönlich gekannt oder aber gemeinsame intellektuelle Räume geteilt hatte, die, inklusive der Kontakte, nach 1945 aufgefrischt und netzwerkartig verdichtet werden konnten. Was nach 1945 zu tun war, glaubte man zu wissen oder zumindest zu ahnen, weil man es schon - im gemeinsamen intellektuellen Raum - in den 1930er und 1940er Jahren gemacht hatte; diesen Raum hatten damals zwei Zeitschriften gebildet, Europäische Revue und Neue Rundschau, die nicht nur viele gemeinsame Autoren gehabt hatten, die man nach 1945 für Merkur gewinnen konnte (außer Curtius waren es mindestens weitere 47 Autoren $^{61}$ ), sondern - eine weitere Gemeinsamkeit - in ihnen hatten als Schriftleiter und Herausgeber eben H. Paeschke, J. Moras und W. von Einsiedel verantwortlich gezeichnet, also der enge Merkur-Kreis.

Bereits diese ersten kursorischen Bemerkungen lassen erahnen, dass jeder Versuch, Merkur aus dem Wechselspiel von Neuanfang und Kontinuität heraus zu interpretieren, nicht nur ästhetische und politische Aspekte mit den jeweiligen intellektuellen Biographien der engeren Kreise der Merkurianer verschränken, sondern - darüber hinaus zwingend auch die intellektuellen Räume, also Netzwerke, auf denen Merkur aufbaute, in ihren Verflechtungen und historisch bedingten Transformationen eben auf Kontinuität und Diskontinuität hin erfassen müsste. Da dies an dieser Stelle kaum zu leisten ist, sei abschließend zumindest in einigen Thesen angedeutet, wie man dieses komplexe Problem enger fokussieren könnte, ohne es allzu sehr zu simplifizieren. Somit, in aller Kürze, zu einem der beiden Merkur-Herausgeber, Joachim Moras.

60 Zum engeren Mitarbeiterkreis gehörten W. von Einsiedel, K. A. Horst, F. Kemp, W. E. Süskind, C. Hohoff, H. E. Holthusen, J. von Kempski. Breitere Kreise der Netzwerke um Merkur wurden gebildet von: M. Boveri, W. Boehlich, A. Gehlen, G. R. Hocke, M. Bense, M. Rychner, C. J. Burckhardt, G. Steinbömer alias G. Hillard, G. Benn, A. Fabri und vielen anderen. Der Grad der Zusammenarbeit lässt sich an der Zahl der Briefe ablesen, die im DLA Marbach aufbewahrt sind.

61 vgl. Kießling, Friedrich (2012): Die undeutschen Deutschen. Eine ideengeschichtliche Archäologie der alten Bundesrepublik 1945-1972, Paderborn, S. 41. 


\section{Joachim Moras: der Mitherausgeber von Merkur}

Der Name Joachim Moras (1902-1961) figurierte im Impressum von Merkur zwar erst ab der ersten Nummer des zweiten Jahrgangs (1948), ${ }^{62}$ doch er war von Anfang an als Herausgeber dabei, mit Hans Paeschke die Konzeption in langen Briefen besprechend und folglich auch umsetzend. Seine uneingestandene Mitarbeit hatte eben mit der Rolle von Moras in der Zeitschrift Europäische Revue zu tun gehabt, die man in Anbetracht der Nachkriegsbedingungen in den französischen Besatzungszonen, wo Merkur zunächst erschien, ${ }^{63}$ für ein zu großes Risiko hielt, was erst die positive Spruchkammerentscheidung im Sommer 1947 ändern konnte. Gerade bei Moras könnte es somit überaus aufschlussreich sein, die Linien nachzuzeichnen zwischen dessen schriftleitenden und verlegerischen Aktivitäten in der Europäischen Revue der 1930er und 1940er Jahre ${ }^{64}$ und dem Merkur, den Moras bis zu seinem Tode 1961 im Duo mit Paeschke herausgab. Europäische Revue, die 1925 unter Karl Anton Rohan durchaus vielversprechend angefangen, allerdings in den 1930er Jahren ihre faschistoiden, europäisch konservativen Akzente immer defensiver zu vertreten imstande war, bis sie - dies gerade in den Jahren, als sie von Moras herausgegeben wurde - zu einem instrumentalisierten Organ der NS-Propaganda wurde, das mit Einverständnis der Herausgeber unter dem Deckmantel der scheinbaren gedanklichen Stabilität ${ }^{65}$ der Zeitschrift die nationalsozialistische Ideologie propagierte, dürfte fürs Erfassen der Kontinuitäten und Diskontinuitäten im Hinblick auf Merkur zumindest genauso ergiebig sein wie Neue Rundschau. Denn diese wurde in den inkriminierten Jahren zunächst 1937-1938 kurz von W. von Einsiedel, der sich bis dahin primär als Übersetzer und Vermittler der englischsprachigen Literatur betätigt hatte, und in den Jahren 1940-1944 von H. Paeschke herausgegeben. Allerdings war die von Moras mitgeprägte Phase in Europäische Revue viel länger als diejenige seiner Merkur-Kollegen in Neue Rundschau, dementsprechend höher der Druck der nationalsozialistischen Direktiven, sowie das Maß an opportunistischem Lavieren, zu dem auszuweichen er bereit war, um die zunehmend schwindenden Enklaven der Freiheit seiner Zeitschrift

62 In den Rückblickpublikationen steht Moras zum Teil stark im Schatten von Paeschke, was ja neben seinem zwangsbedingt verspäteten Start auch dem geschuldet sein dürfte, dass Paeschke Merkur nach dem Tode von Moras weitere 17 Jahre im Alleingang herausgab. Ob der als „schöngeistige“ apostrophierte Moras in Merkur tatsächlich der zweite Mann war, müsste erst ermittelt werden. vgl. Bohrer, Karl Heinz - Scheel, Kurt (1997): Die Botschaft des Merkur. Eine Anthologie aus fünfzig Jahren der Zeitschrift. Stuttgart: Klett Cotta, S. 8.

63 Die ersten neun Nummern sind in Heller und Wegner Verlag in Baden Baden erschienen, ab der 10. Nummer (1948) wechselte man zur Deutschen Verlagsanstalt nach Stuttgart.

64 Hier war Moras von 1933 als Schriftleiter, seit 1938 als Herausgeber tätig, dies bis 1943.

65 Vgl. dazu eine interne Mitteilung aus dem Jahre 1934:

„Während allen übrigen Organen ähnlicher Art die Gleichschaltung an die Stirn geschrieben ist, wird bei der ,Europäischen Revue‘ nur der bis in alle Einzelheiten orientierte Leser eine gewisse Anpassung an die politische Gesamtsituation verspüren. Für weniger feine Augen wird „Europäische Revue` heute dasselbe sein wie vor Jahren [...] Je unauffälliger die Zeitschrift als Propagandaorgan eingesetzt werde, umso nützlichere Arbeit kann die ,Europäische Revue‘ leisten.“ Werbungsplan für die Europäische Revue vom 24. 1. 1937, im Nachlass von Lilly von Schnitzler, zit. nach: Müller, Guido (2005): Europäische Gesellschaftsbeziehungen nach dem Ersten Weltkrieg. Das Deutsch-Französische Studienkomitee und der Europäische Kulturbund. München: Oldenbourg, S. 404. 
doch noch zu bewahren. ${ }^{66}$ Alles in allem, Moras opportunistische Bereitschaft, sich auf die Bedingungen des NS-Propagandaministeriums einzulassen, um davon für seine Zeitschrift zu profitieren ${ }^{67}$ war offenbar nicht zu vergleichen mit dem auf tröstendes und verdecktes Schreiben hin ausgerichteten Zwangsprogramm der Neuen Rundschau zu von Einsiedels und Paeschkes Zeiten. Im Merkur allerdings, und dies scheint zentral, wusste man um das $\mathrm{Maß}$ der Kompromittierung, weshalb man Moras zunächst zurückhielt und beim Anwerben der Beiträger immer strategisch abwog, ob seine Anwesenheit die eventuelle Zusammenarbeit nicht vereiteln würde, zugleich wusste man sich aber seine Kontakte in höheren politischen, industriellen aber auch künstlerischen Etagen aus den früheren aber auch späteren Phasen der Europäischen Revue zunutze zu machen. Es war nicht nur der aristokratisch-großindustrielle Background dieser Zeitschrift, von dessen Kapital Moras einiges in die Nachkriegszeit hinüberretten konnte, es waren auch die intellektuellen Sympathien für eine europäisch angedachte deutsch-französische Versöhnungsarbeit, der sich Europäische Revue lange Jahre verschrieben hatte, aus der dann etliche für Merkur nützliche Kontakte resultierten, zumal einige dieser Intellektuellen Moras auch aus den NS-Jahren als einen der wenigen Anständigen in Erinnerung hatten und ihm doch verbunden gewesen sein dürften (T. Heuss, D. Sternberger, M. Bense, R. Kassner, R. A. Schröder, O. von Taube u. a.).

Diesem für den Start von Merkur unumgänglichen Kapital an Kontakten arbeitete auch Paeschke entgegen, sofern er seine überaus zwielichtigen Jahre eines „former counter-espionage agent who had been active with the German military defence division in southern France during the later war years “68 in gute Nachkriegswährung umzumünzen wusste, denn es gelang ihm unmittelbar nach dem Krieg, das Vertrauen der Franzosen zu gewinnen, um - gemeinsam mit Gerhard Heller, einer noch ambivalenteren Persönlichkeit der deutsch-französischen Beziehungen ${ }^{69}$ - die deutschsprachige Zeitschrift Lancelot. Ein Bote aus Frankreich herausgeben zu können. Hier redigierte und übersetzte

66 Um das Überleben der Zeitschrift zu garantieren, scheint Moras in der Tat massiv laviert zu haben, „Gruppenbildungen innerhalb des staatlichen und parteiamtlichen Überwachungsapparats ausnutzend“. Vgl. Pross [Anm. 9], S. 127. Die offiziellen Artikel nahmen im Laufe der Zeit zu, neue die politische Parteilinie unterstützende Rubriken wurden gegründet, die Zahl der parteikonformen Autoren stieg, während der Teil, der Moras übrig blieb, immer geringer wurde, sich vielmehr nur auf Rezensionen beschränkte, wobei immer weniger rezensiert, stattdessen nur noch über Bücher berichtet wurde. Dass es darin hie und da gelang, eine gewisse Freiheit in der Wahl der präsentierten Bücher sowie der Rezensenten zu bewahren, kann freilich nicht in Abrede gestellt werden.

67 Moras war offenbar auch etwa im internationalen Netzwerk Europäische Schriftsteller Vereinigung aktiv, einer Anti-Pen Organisation, die unter NS-Aufsicht die restliche Welt von besten europäischen Absichten der nationalsozialistischen Ideologie überzeugen sollte, eine propagandistisch sehr wohl kalkulierte Strategie. Moras nahm offenbar an der Weimarer Tagung im Jahre 1941 teil. Vgl. Bailey [Anm. 44], S. 45.

68 Bailey [Anm. 44], S. 26.

69 G. Heller, in dessen Verlag die ersten neun Nummern von Merkur erschienen, hat während des Krieges im besetzten Frankreich für deutsche Propaganda gearbeitet, er war zuständig für die Literaturpolitik der Besatzungsmacht, und konnte darum neben Verboten auch Erlaubnisse anordnen. Nach dem Krieg dürften vor allem die Erlaubnisse (und sehr wohl auch gute Kontakte) dazu beigetragen haben, dass Heller relativ bald im kulturellen Bereich Fuß fassen konnte. Mit Erlaubnis der französischen Besatzungsmacht gab er - gemeinsam mit Jacqueline Grappin - kurz Lancelot. Briefe aus Frankreich heraus, stellte dem Merkur für die ersten neun Nummern seinen Verlag zur Verfügung, um 1950 den Stahlberg-Verlag in Karlsruhe mit zu gründen, in dem 
Paeschke ein Jahr lang (1946) Texte französischer Humanisten (L. Aragon), dann verwarf er sich mit der französischen Redaktion, was er allerdings verschmerzen konnte, denn er gewann französische Lizenz, um 1947 Merkur zu gründen. Die derart ineinanderübergehenden und in ihrer Verflechtung kaum überschaubaren Netzwerke bildeten in ihren dynamischen Transformationen an der Schwelle zwischen der Kriegszeit und den beiden Nachkriegszeiten für Merkur die Grundlage, die der Zeitschrift sowohl zum Leben verholfen, ihr dasselbige aber zugleich schwer gemacht hat. Denn die Kontinuitäten im personellen aber auch institutionellen Sinne waren zwar für die Zeitschrift existentiell unabdingbar, doch sie standen deren vollen Unabhängigkeit im Wege. Am Profil der Zeitschrift haben sie deutliche Spuren hinterlassen, insofern sich darin - zumindest bis 1949 - nicht nur der Druck der Alliierten Besatzungskulturpolitik, sondern - darüber hinaus - auch Einfluss einiger Persönlichkeiten mit aristokratischem, diplomatischem, großindustriellem oder kulturpolitischem Hintergrund bemerkbar machte, deren Texte man in Merkur vor allem aus ökonomisch- oder symbolisch-pragmatischen Gründen veröffentlicht hat. Noch massiver mag sich der Druck der Verlagskontinuitäten ausgewirkt haben, mussten doch beide Herausgeber in ihrem Kampf um Unabhängigkeit von Merkur stets ihre Interessen gegen den Druck der Deutschen Verlagsanstalt behaupten, eines Verlagshauses, das zwar eine gewisse ökonomische Sicherheit garantierte, aber als ehemaliger NSDAP-Verlag, in dem ab 1934 Europäische Revue mit Moras als Herausgeber wie auch Deutsche Zukunft (hier war Paeschke 1937-39 Redaktionsmitglied) herausgegeben worden waren, beide Herausgeber an dunklere Jahre ihres Lebens erinnert haben dürfte.

Daher die oft aufgekommenen Befürchtungen ums Überleben der Zeitschrift, die in diverse, teils verzweifelte Versuche um Spendenaktionen mündeten, ${ }^{70}$ aber auch etliche Parallelaktivitäten von Moras, dem die finanzielle Unsicherheit offenbar mehr als Paeschke zusetzte. Symptomatisch an allen diesen abseits der Grenzen von Merkur geführten Projekten von Moras ist indes, dass sie im Endeffekt dem Merkur zuträglich waren. Die persönlichen Interessen mündeten letztendlich in die Interessen der Zeitschrift, sind daher als integraler Bestandteil der Netzwerkanalysen zu betrachten, die Merkur aus dem Spannungsfeld der Kontinuitäten und Diskontinuitäten herausinterpretieren. So war es im Falle eines überaus ambitionierten Projektes der rowohlts deutschen enzyklopädie, an dessen Konzeption sich Moras offiziell beteiligt hatte: Dieses enzyklopädische Projekt wurde von 1955 an ca. zehn Jahre lang im Rowohlt Verlag vom italienischen Humanismuskenner Ernesto Grassi mit dem Ziel realisiert, die modernen Natur-, Kultur-, Geistes- und Gesellschaftswissenschaften im Taschenbuchformat, also billig und daher einer breiten Öffentlichkeit, zugänglich zu machen. Moras Rolle bei diesem ob der Auflagen- und Verkaufszahlen den westdeutschen Büchermarkt der 1950er Jahre wissenschaftlich revolutionierenden Unternehmen ${ }^{71}$ bestand darin, zwischen 15. 4. 1954 und

er insbesondere für die Übersetzungen aus romanischen Sprachen zuständig war. Vor allem bezüglich der romanischen Literaturen war Heller für Merkur einer der wichtigsten Vermittler.

70 vgl. den im Jahre 2004 publizierten Briefwechsel zwischen Gottfried Benn und den Merkur-Herausgebern Hans Paeschke und Joachim Moras, herausgegeben von Holger Hof. Stuttgart: Klett Cotta, S. $50 \mathrm{ff}$.

71 vgl. Döring, Jörg - Lewandowski, Sonja - Oels, David (2017): Wissenschaft im Taschenbuch 1955-1968. Hannover: Wehrhahn, S. 17. 
1. 1. 1955 mit dem in Südamerika verweilenden Grassi über die Reihe zu korrespondieren, ihn bei den Gesprächen mit den Autoren zu vertreten, ja ihm, angefangen mit dem ersten Band, also vom 1. Januar 1955 bis zum Jahresende, als Herausgeber zu assistieren, wobei man Moras in Aussicht gestellt hat, „auch für die Zukunft als Assistent von Herrn Professor Grassi beim weiteren Ausbau dieser Reihe“72 seine Arbeit für den Rowohlt Verlag fortsetzen zu können. Dazu ist es zwar nicht mehr gekommen, denn Moras musste nach der neunmonatigen Arbeit für Grassi aufgrund seiner Lektoratsarbeit bei DVA die Abmachungen betreffend der Zusammenarbeit über den 1. Januar 1955 hinaus rückgängig machen, doch bereits die Korrespondenz zwischen Moras, Grassi und Ledig-Rowohlt sowie der Blick auf die Namen, Titel, Themen und Akzente dieser Reihe, die sich nicht selten mit den Merkurschen überlappen, lässt erahnen, wie aufschlussreich es sein könnte, Moras innerhalb des dynamisch interagierenden Spannungsfeldes der Netzwerke um Merkur und rowohlts deutsche enzyklopädie zu situieren. Am ehesten kann man sich dies als eine kooperierende Konkurrenz vorstellen, zumal diese Zusammenarbeit mit Vorabdrucken der enzyklopädischen Titel im Merkur, reziproken Werbeverweisen sowie diverser Formen der Beratungen seitens Moras weit über die Vertragsfrist hinaus andauerte, wie etwa die auf Moras Initiative zurückzuführende Präsenz von G. R. Hocke oder M. Boveri in rde oder einige in Merkur abgedruckte Kapitel der in $r d e$ publizierten Texte von F. Altheim, H. Schelsky u. A. belegen.

Im zweiten wichtigen Parallelprojekt, an dem sich Moras mitunter als Herausgeber in den Jahren 1954-1961 beteiligte, den vom Kulturkreis im Bundesverband der deutschen Industrie im Jahrestakt herausgegebenen als Jahresring betitelten Bestandsaufnahmen, die, so der Untertitel, „Querschnitt durch die deutsche Literatur und Kunst der Gegenwart" präsentierten, scheint er an seine in Europäische Revue erprobte einstige Rolle des pragmatisch agierenden Vermittlers zwischen Kunst und (Groß)Industrie angeknüpft und diese erweitert zu haben. Moras durfte sich hier maßgeblich an den kanonisierenden Jahresüberblickprozessen beteiligen, indem er die Auswahl nicht nur der hier abgedruckten Texte mitbeeinflusste, sondern auch den als jeweilige Preisträger des Kulturkreises ausgezeichneten Autoren eine nicht unbeträchtliche finanzielle Förderung zukommen ließ. An der Liste der hier vertretenen AutorInnen und PreisträgerInnen fallen zumindest drei Sachen auf: Nicht überraschend kommen auch hier wiederum Namen und Texte vor, denen man haufenweise zeitgleich auf den Seiten von Merkur begegnet, ab und zu werden sogar identische Texte abgedruckt. Auch hier ist wie im Merkur eine Tendenz festzumachen, die bewährten Namen der Zwischenkriegszeit über die zu der Zeit doch schon massiv aufkommenden Vertretern der jungen Generation nicht zu vernachlässigen, was manche im Hinblick auf die Karrieren in der NS-Zeit problematische Fälle (L. Ziegler, G. Gaiser, E. Jünger, F. Sieburg), wenn nicht bedenkliche Fehltritte (F. Tumler, W. Emrich) nach sich zog. Diese Zwischenposition kommt hier auch darin zum Tragen, dass Jahresring, darin nicht unähnlich dem Merkur, als ein privilegiertes Forum der Zwischengeneration (mittlere Generation) daherkommt, die ihren intakten Anschluss an die Zwischenkriegszeitmoderne nach 1945 zur Rolle der lebendigen und

72 Heinrich Maria Ledig-Rowohlt an Joachim Moras, 26. 3. 1954. DLA Marbach. 
durch die NS-Zeit nur unkompromittiert geglaubten Vermittler und Türöffner ${ }^{73}$ für die jüngeren Generationen umzumünzen wusste. Dass sich Moras mit dieser Rolle gerne identifizierte, ja sie als eine kollektive Rolle für die Merkurianer insgesamt aufgefasst haben mochte, sieht man am letzten hier zu erwähnenden seiner Projekte, dem vom Stifterverband und der Rockefeller Foundation geförderten, bilanzierenden Band Deutscher Geist zwischen gestern und morgen, den Moras 1954 gemeinsam mit Paeschke und unter Mitwirkung von W. von Einsiedel herausgegeben hat. Die hier in vielen Studien beklagte fehlende Mitte bezog sich nicht nur auf geopolitische (Deutschland als das einstige Land der Mitte, dem die Mitte nun abhandengekommen ist) oder soziale (fehlende Mittelschicht) Aspekte, sondern einfach auch auf die mittlere Generation, deren Absenz als fatal schien, und somit gerade durch die „in einem zeitlichen Radius von etwa 10 Jahren - um die Jahrhundertwende herum Geborenen“74 angehalten und wettgemacht werden sollte. Was hier von Einsiedel (geb. 1903) im Jahre 1954 als Programm formulierte, agierte Moras (geb. 1902) im Jahresring in den Jahren 1954-61 und gemeinsam mit Paeschke (geb. 1911) bereits seit 1947 im Merkur aus, und dass dieser Aspekt auch bei der von Grassi (geb. 1902) herausgegebenen rde (1955-1968) mit im Spiel gewesen sein dürfte, scheint ob etlicher Überlappungen nicht ganz ausgeschlossen zu sein.

Aus all diesen kursorischen Ausführungen ergeben sich freilich noch keine stichhaltigen Antworten, allenfalls ein geringfügiger Befund, der allerdings zu denken geben könnte: Die einst bahnbrechende Aufforderung A. Schildts, etliche in der zweiten Hälfte der 1950er Jahre umgesetzten Projekte auf ihre - unter welch konservativen Auspizien auch immer sich abspielende - Modernität hin zu untersuchen, hat unter anderem dazu geführt, dass der früher bruchartig in die 1960er Jahre gelegte Modernisierungswandel nun vielmehr als ein längerfristiger Prozess aufgefasst wird, dessen Anfang eben um das Jahr 1955 situiert wird. Dass demzufolge dann - mittlerweile konsenshaft - von langen 1960er Jahren die Rede ist, deren Anfang in das letzte Drittel der 1950er Jahre vorverlegt wird, erscheint plausibel, wie auch die dadurch um einiges kürzer gewordenen 1950er Jahre, die sich somit zwischen 1949 und 1955 (oder 1957 55 ) eben noch unter ganz unmodernen Bedingungen der konservativen Integrationen abgespielt haben sollen. Eine sehr wohl netzwerkartig angelegte, detaillierte Analyse des Falles Joachim Moras im Spannungsfeld der Kontinuität und Diskontinuität, dessen ineinanderübergehende Projekte ja von 1947 bis 1961 reichten, könnte allerdings den Blick auf die inkriminierten Jahre wohl noch genauer disponieren, sofern sie ermitteln könnte, was genau uns eventuell das Recht gibt, zwischen rde, Jahresring und Merkur eine Zäsur zu legen. Moras wäre somit für uns ein Grund, nach der Zäsur des Jahres 1945 auch die Zäsur des Jahres 1955 mit einem Fragezeichen zu versehen.

73 vgl. Klessinger, Hanna (2011): Bekenntnis zur Lyrik: Hans Egon Holthusen, Karl Krolow, Heinz Piontek und die Literaturpolitik der Zeitschrift Merkur in den Jahren 1947 bis 1956. Göttingen: Wallstein, S. 101.

74 Einsiedel, Wolfgang von (1954): Land ohne Mitte. In: Moras, Joachim - Paeschke, Hans - Einsiedel, Wolfgang von (hrsg.): Deutscher Geist zwischen gestern und morgen. Stuttgart: DVA, S. 438.

75 So in: Hodenberg, Christina (2006): Konsens und Krise. Eine Geschichte der westdeutschen Medienöffentlichkeit 1945-1973. Göttingen: Wallstein. 


\section{Quellen und Literatur}

Bailey, Christian (2013): Between Yesterday and Tomorrow: German Visions of Europe, 19261950. New York - Oxford: Berghahn.

Bock, Hans Manfred (2001): Die fortgesetzte Modernisierung des Konservativismus. Merkur: Deutsche Zeitschrift für europäisches Denken 1947-1957. In: Grunewald, Michel - Bock, Hans Manfred (hrsg.): Der Europadiskurs in den deutschen Zeitschriften (1945-1955). Bern: Peter Lang, S. 149-185.

Bock, Hans Manfred (1990): Die Politik des ,Unpolitischen‘. Zu Ernst Robert Curtius' Ort im politisch-intellektuellen Leben der Weimarer Republik. In: lendemains. Études comparées sur la France 59, S. 16-61.

Bohrer, Karl Heinz - Scheel, Kurt (1997): Die Botschaft des Merkur. Eine Anthologie aus fünfzig Jahren der Zeitschrift. Stuttgart: Klett Cotta.

Curtius, Ernst Robert (1949): Ortega y Gasset. In: Merkur 5, S. 417-429. Später abgedruckt in: ders. (1954): Kritische Essays zur europäischen Literatur. Bern, S. 266-280.

Doering-Manteuffel, Anselm (1993): Die Kultur der 50er Jahre im Spannungsfeld von „Wiederaufbau“ und „Modernisierung“. In: Schildt, Axel - Sywottek, Arnold (hrsg.): Modernisierung im Wiederaufbau. Die westdeutsche Gesellschaft der 50er Jahre. Bonn: Dietz, S. 533-540.

Döring, Jörg - Lewandowski, Sonja - Oels, David (2017): Wissenschaft im Taschenbuch 1955-1968. Hannover: Wehrhahn.

Einsiedel, Wolfgang von (1954): Land ohne Mitte. In: Moras, Joachim - Paeschke, Hans - Einsiedel, Wolfgang von (hrsg.): Deutscher Geist zwischen gestern und morgen. Stuttgart: DVA, S. 433-440.

Enzensberger, Hans Magnus (1988): Mittelmaß und Wahn. Frankfurt am Main: Suhrkamp.

Fischer, Ludwig (1993): Zur Sozialgeschichte der westdeutschen Literatur. In: Schildt, Axel Sywottek, Arnold (hrsg.): Modernisierung im Wiederaufbau. Die westdeutsche Gesellschaft der 50er Jahre. Bonn: Dietz, S. 551-562.

Hodenberg, Christina (2006): Konsens und Krise. Eine Geschichte der westdeutschen Medienöffentlichkeit 1945-1973. Göttingen: Wallstein.

Kiesel, Helmuth (1997): Die Restaurationsthese als Problem für die Literaturgeschichtsschreibung. In: Erhart, Walter - Niefanger, Dirk (hrsg.): Zwei Wendezeiten. Blicke auf die deutsche Literatur 1945 und 1989. Tübingen: Max Niemeyer, S. 13-46.

Kießling, Friedrich (2014): Fruchtbare Zerrissenheit. Der Merkur in der frühen Bundesrepublik. In: Zeitschrift für Ideengeschichte 1, S. 87-100.

Kießling, Friedrich (2012): Die undeutschen Deutschen. Eine ideengeschichtliche Archäologie der alten Bundesrepublik 1945-1972. München - Wien - Zürich: Schöningh.

Kießling, Friedrich (2011): „Gesprächsdemokraten“ - Walter Dirks` und Eugen Kogons Demokratie- und Pluralismusbegründungen in der frühen Bundesrepublik. In: Gallus, Alexander - Schildt, Axel (hrsg.): Rückblickend in die Zukunft. Politische Öffentlichkeit und intellektuelle Positionen in Deutschland um 1950 und um 1930. Göttingen: Wallstein, S. 385-412.

Heinrich Maria Ledig-Rowohlt an Joachim Moras, 26. 3. 1954. DLA Marbach.

Moras, Joachim - Paeschke, Hans (1954): Nummer 100. In: Merkur 100, S. 504.

Moras, Joachim - Paeschke, Hans (1948): An unsere Leser. In: Merkur 10, S. 481-484.

Moras, Joachim - Paeschke, Hans - Einsiedel, Wolfgang von (1954): Deutscher Geist zwischen gestern und morgen. Stuttgart: DVA.

Müller, Guido (2005): Europäische Gesellschaftsbeziehungen nach dem Ersten Weltkrieg. Das 
Deutsch-Französische Studienkomitee und der Europäische Kulturbund. München: Oldenbourg.

Oels, David (2013): Rowohlts Rotationsroutine. Markterfolge und Modernisierung eines Buchverlags vom Ende der Weimarer Republik bis in die fünfziger Jahre. Essen: Klartext.

Paeschke, Hans (1978): Kann keine Trauer sein. In: Merkur, 367, S. 1169-1193.

Paeschke, Hans (1963): In Sachen Merkur. In: Merkur 179, S. 1-10.

Parker, Stephen - Davies, Peter - Philpotts, Matthew (2004): The Modern Restoration. Re-thinking German Literary History 1930-1960. Berlin - New York: De Gruyter.

Payk, Marcus (2008): Der Geist der Demokratie. Intellektuelle Orientierungsversuche im Feuilleton der frühen Bundesrepublik: Karl Korn und Peter de Mendelssohn. München: Oldenbourg.

Pross, Harry (1963): Literatur und Politik. Geschichte und Programme literarisch-politischer Zeitschriften im deutschen Sprachgebiet seit 1870. Olten: Walter.

Richter, Hans Werner (1976): Warum schweigt die junge Generation? [Der Ruf, Nr. 2 / 2. September 1946]. In: Neunzig, Hans A. (hrsg.): Der Ruf. Unabhängige Blätter für die junge Generation. München: Nymphenburg, S. 60-65.

Richter, Hans Werner (1962): Zwischen Freiheit und Quarantäne. In: ders. (hrsg.): Bestandsaufnahme. Eine deutsche Bilanz 1962. München: Kurt Desch, S. 11-28.

Schildt, Axel (1999): Zwischen Abendland und Amerika. Studien zur westdeutschen Ideenlandschaft der 50er Jahre. München: Oldenbourg.

Schöning, Matthias (2015): „Zäsur“. Probleme einer historiographischen Angewohnheit. In: Meierhofer, Christian - Wörner, Jens (hrsg.): Materialschlachten. Der Erste Weltkrieg und seine Darstellungsressourcen in Literatur, Publizistik und populären Medien 1899-1929. Osnabrück: Universitätsverlag, S. 67-82.

Schütz, Erhard (2019): Mediendiktatur Nationalsozialismus. Heidelberg: Winter.

Urválek, Aleš (2020): „Diagonallinie der goldenen Mitte“ zwischen Eigenem und Fremdem. Konstellationen der frühnachkriegsdeutschen Internationalisierung am Beispiel von Merkur. Deutsche Zeitschrift für europäisches Denken. In: Hehl, Michael Peter - Tommek, Heribert (hrsg.): Transnationale Akzente. Zur vermittelnden Funktion von Literatur- und Kulturzeitschriften im Europa des 20. Jahrhunderts. Berlin: Peter Lang.

Veit, Walter (1981): Die Stunde Null im Spiegel einiger zeitgenössischer Zeitschriften. In: Hüppauf, Bernd (hrsg.): Die Mühen der Ebenen. Kontinuität und Wandel in der deutschen Literatur und Gesellschaft 1945-1949. Heidelberg: Winter, S. 195-232.

Vormweg, Heinrich (1981): Deutsche Literatur 1945-1960: Keine Stunde Null. In: Durzak, Manfred (hrsg.): Deutsche Gegenwartsliteratur. Ausgangspositionen und aktuelle Entwicklungen. Stuttgart: Metzler, S. 13-31.

Widmer, Urs (1966): 1945 oder die „neue Sprache“. Studien zur Prosa der „Jungen Generation“. Düsseldorf: Schwann. 
Doc. Mgr. Aleš Urválek, Ph.D. / urvalek@phil.muni.cz

Masarykova univerzita, Filozofická fakulta, Ústav germanistiky, nordistiky a nederlandistiky Arna Nováka 1, 60200 Brno, CZ

This work can be used in accordance with the Creative Commons BY-SA 4.0 International license terms and conditions (https://creativecommons.org/licenses/by-sa/4.0/legalcode). This does not apply to works or elements (such as image or photographs) that are used in the work under a contractual license or exception or limitation to relevant rights 
